\title{
Sobrevivência de plântulas, características fotossintéticas e crescimento de Discocarpus pedicellatus (Phyllanthaceae)
}

Seedling survival, photosynthetic characteristics and growth of Discocarpus pedicellatus (Phyllanthaceae)

\author{
Dinara de Andrade Mercês ${ }^{1}$, Marayana Prado Pinheiro ${ }^{2}$, Josafá Amaral de Oliveira Filho ${ }^{1}$, Solange França ${ }^{3}$, \\ Fábio Pinto Gomes ${ }^{1}$, Pedro Fiaschi ${ }^{4} \&$ Marcelo Schramm Mielke ${ }^{1,5}$
}

\begin{abstract}
Resumo
Discocarpus pedicellatus é uma espécie arbórea endêmica da Mata Atlântica que apresenta regeneração natural por meio da formação de bancos de plântulas, ocorrendo em populações com manchas de elevada densidade de árvores adultas nas florestas semidecíduas do sul da Bahia. Foram realizados dois estudos, um no campo e outro em casa de vegetação, visando analisar a sobrevivência de plântulas e os efeitos do sombreamento nas características fotossintéticas e no crescimento de plantas jovens de $D$. pedicellatus. No início das avaliações no campo a densidade de plântulas foi muita elevada, em torno de 700 plântulas $\mathrm{m}^{-2}$. Ao final de dois anos esses valores variavam entre $5 \mathrm{e}$ 190 plântulas $\mathrm{m}^{-2}$, sendo raros os indivíduos que apresentavam folhas verdadeiras. Em casa de vegetação, os valores da taxa fotossintética líquida em saturação de radiação luminosa $\left(\mathrm{A}_{\max }\right)$, da irradiância de compensação (Ic), da taxa de respiração no escuro (Rd), da taxa de crescimento relativo (TCR) e da taxa assimilatória líquida (TAL), indicam que essa espécie apresenta crescimento lento, típico de espécies que regeneram no interior de florestas com dossel fechado. Além disso, com o aumento do sombreamento observou-se uma diminuição significativa da Rd, indicando que a sobrevivência no sub-bosque está associada com baixas taxas metabólicas e de crescimento. Os resultados obtidos indicam que a estratégia de regeneração por bancos de plântulas e a capacidade de ajustar o seu metabolismo em função da radiação luminosa disponível são fatores importantes para o estabelecimento de populações de $D$. pedicellatus com manchas de elevada densidade populacional nas florestas semidecíduas do sul da Bahia.
\end{abstract}

Palavras-chave: Mata Atlântica, fotografias hemisféricas, tolerância a sombra.

\begin{abstract}
Discocarpus pedicellatus is an endemic tree species in the Atlantic Forest. It presents natural regeneration from seedling banks and populations with patches of high-density of adult trees in the semideciduous forests of southern Bahia, Brazil. Two studies were conducted, in the field and under greenhouse conditions, aiming to analyze seedling survival and the effects of shade on growth and on photosynthetic characteristics of $D$. pedicellatus saplings. In the field, the density of seedlings was very high at the beginning of the study, in some cases exceeding 700 seedlings $\mathrm{m}^{-2}$. After two years of observations, density values were between 5 and 190 seedlings $\mathrm{m}^{-2}$ and few individuals showed the first true leaves. In greenhouse, the results obtained for light-saturated net photosynthetic rate $\left(\mathrm{A}_{\max }\right)$, the compensation irradiance (Ic), the dark respiration rate (Rd), the relative growth rate (TCR) and the net assimilation rate (TAL), suggest that this species presents slow growth, typical of species that regenerate in the understory of mature forests under closed canopies. Also, in response to increase in shading there was a significant decrease in $\mathrm{Rd}$, indicating that the survival in the forest understory may be associated to low metabolic and growth rates. The results indicate that the regeneration strategy by seedling banks and the ability to adjust their metabolism in response to the low available light radiation are important factors that explain the establishment of high-population density patches of $D$. pedicellatus in the semideciduous forests of southern Bahia, Brazil. Key words: Atlantic rainforest, hemispherical photographs, shade tolerance.
\end{abstract}

\footnotetext{
${ }^{1}$ Universidade Estadual de Santa Cruz - UESC/DCB, Depto. Ciências Biológicas, Rod. Ilhéus-Itabuna, km 16, 45650-000, Ilhéus, BA, Brasil.

${ }^{2}$ Universidade Estadual do Sudoeste da Bahia - UESB/DCN, Depto. Ciências Naturais, Est. do Bem-Querer, km 4, 45083-900, Vitória da Conquista, BA, Brasil.

${ }^{3}$ Universidade Estadual de Santa Cruz - UESC/DCAA, Depto. Ciências Agrárias e Ambientais, Rod. Ilhéus-Itabuna, km 16, 45650-000, Ilhéus, BA, Brasil.

${ }^{4}$ Universidade Federal de Santa Catarina, Depto. Botânica (CCB), Campus Universitário, Trindade, 88040-900, Florianópolis, SC, Brasil.

${ }^{5}$ Autor para correspondência: msmielke@uesc.br
} 


\section{Introdução}

A Mata Atlântica do sul da Bahia figura entre as áreas de maior riqueza de espécies arbóreas do mundo (Martini et al. 2007). De acordo com Thomas et al. (1998) entre 45 e 48\% das espécies de plantas vasculares desta região são endêmicas da Mata Atlântica, cerca de 7,5\% apresentam distribuição disjunta entre a Mata Atlântica da Bahia e a Amazônia, e entre 25 e $30 \%$ são encontradas exclusivamente nas florestas ombrófilas litorâneas do norte do Espírito Santo ao sul do estado da Bahia. Nesse contexto, a região de transição entre as florestas ombrófilas do litoral e as florestas estacionais do interior do estado da Bahia abriga áreas bastante fragmentadas, mas ainda pouco conhecidas floristicamente (Thomas et al. 2009). A Reserva Particular do Patrimônio Natural (RPPN) da Serra do Teimoso é um dos fragmentos mais representativos da transição entre as florestas ombrófilas litorâneas e as florestas estacionais. A RPPN Serra do Teimoso está localizada na fazenda de mesmo nome e corresponde a uma mata de encosta variando de 200 a 850 m.s.m. Nas menores altitudes da reserva (entre 200 e 500 m.s.m.) predominam espécies típicas de floresta estacional semidecidual, devido às variações sazonais do regime pluviométrico, enquanto no topo (acima de 500 m.s.m.) predominam espécies de florestas ombrófilas, onde a sazonalidade climática é menos intensa devido à retenção de umidade orográfica (Amorim et al. 2005).

Discocarpus pedicellatus Fiaschi \& Cordeiro (Phyllanthaceae) é a espécie mais comum nas áreas de terras baixas da Serra do Teimoso, com registro de 90 indivíduos numa área de apenas ca. 0,3 ha (Thomas et al. 2009). Apesar de ser uma espécie muito comum na RPPN Serra do Teimoso, onde forma manchas com elevada densidade populacional, existem poucas populações conhecidas da espécie, a qual possui distribuição restrita às florestas estacionais semideciduais do sul da Bahia (Fiaschi \& Cordeiro 2005). Além de ocorrer na área da RPPN Serra do Teimoso, a espécie é também conhecida apenas na região de Itarantim, próximo à divisa da Bahia com Minas Gerais (Fiaschi \& Cordeiro 2005). Discocarpus pedicellatus é uma espécie dióica, cujos indivíduos adultos apresentam porte médio (até cerca de $20 \mathrm{~m}$ de altura) e a regeneração ocorre pela formação de bancos de plântulas, formados a partir de eventos de frutificação em massa com intervalos de cinco a dez anos.
Estudos sobre a regeneração natural de espécies arbóreas são de grande importância para a compreensão dos processos ecológicos relacionados com a dinâmica florestal e o manejo adequado de fragmentos florestais (Higuchi et al. 2006). Além disso, tais estudos podem contribuir para a produção de mudas de espécies ameaçadas, por meio da coleta de plântulas germinadas no campo (Auer \& Graça 1995; Viani \& Rodrigues 2007). A regeneração natural por espécies que apresentam a formação de bancos de plântulas em ambientes florestais pode ser afetada por vários fatores, como a proximidade das árvores matrizes (Comita \& Hubbel 2009; Li et al. 2009), a sazonalidade climática (Moraes \& Paoli 1999; Marimon et al. 2012), a formação de clareiras no dossel florestal (Marimom et al. 2012), entre outros.

Em comparação com áreas abertas e clareiras, no interior das florestas com dossel fechado há uma diminuição da disponibilidade de radiação luminosa, das temperaturas do ar e do solo, da entrada de precipitação e da disponibilidade de nutrientes, além de um aumento da umidade relativa e consequente redução do déficit de pressão de vapor do ar (Lüttge 2008). A penetração direta e intermitente da luz ao longo do perfil do dossel desempenha um papel extremamente importante na regeneração natural e na produtividade primária das plantas que se desenvolvem na sombra de matas fechadas (Chazdon 1988; Leakey et al. 2005). Visto que a posição ocupada pelas diferentes espécies na sucessão florestal está relacionada com a habilidade de plântulas e indivíduos jovens para sobreviver e crescer em função das condições microambientais prevalecentes (Lüttge 2008), o grau de tolerância à sombra é reconhecidamente um fator de grande importância na determinação do estágio sucessional de espécies arbóreas tropicais (Souza \& Válio 2003). Além disso, diversos estudos têm indicado que as estratégias que permitem as plântulas e indivíduos jovens sobreviverem e crescerem no sub-bosque parecem estar relacionadas com a fisionomia florestal (Kitajima 1994; Walters 1999; Poorter 2009). Segundo Poorter (2009), em florestas tropicais úmidas o crescimento e a sobrevivência em ambientes sombreados estão relacionados com a capacidade de alocação de carbono para tecidos de armazenamento e defesa, enquanto que nas florestas estacionais haveria uma tendência à maximização da interceptação da radiação luminosa associada com diminuições nas taxas respiratórias. 
A luz é a principal fonte de energia para a assimilação de carbono (Valladares \& Niinemets 2008), afetando estabelecimento de plântulas e o crescimento nos ambientes sombreados do sub-bosque florestal (Kelly et al. 2009; Kenzo et al. 2011). Do ponto de vista da utilização da luz como um recurso do ambiente físico, a adaptação ou a aclimatação de plantas a ambientes de baixa ou elevada disponibilidade de radiação luminosa pode ocorrer em níveis bioquímicos, celulares ou de planta inteira. Folhas de sombra têm uma maior proporção de parênquima paliçádico, maior teor de clorofila, menor razão clorofila a/b e mais nitrogênio alocado para os sistemas coletores de luz, quando comparadas com folhas de sol (Evans \& Poorter 2001). Em escala de planta inteira, em baixa disponibilidade de radiação luminosa a maioria das espécies apresenta a tendência de alocar mais biomassa para a parte aérea do que para as raízes, aumentando a razão de área foliar (Evans \& Poorter 2001; Valladares \& Niinemets 2008; Niinemets 2010). O nível em que uma determinada espécie pode aclimatar-se em ambientes onde a disponibilidade de radiação luminosa é um recurso limitante também está relacionado à sua capacidade de manter um balanço de carbono positivo, proporcionado, entre outros aspectos, por baixas taxas respiratórias (Baltzer \& Thomas 2007; Valladares \& Niinemets 2008). Assim, avaliações do crescimento, em nível de planta inteira, e da fotossíntese, em nível de folhas, podem ser úteis para determinar o nível de tolerância à sombra de espécies florestais (Kelly et al. 2009; Kenzo et al. 2011).

Levando em conta a restrita distribuição geográfica dessa espécie, e a sua importância na composição florística das florestas semideciduais onde está inserida a RPPN Serra do Teimoso (Thomas et al. 2009), foram realizados dois experimentos independentes com o objetivo geral de avaliar a influência da disponibilidade de radiação luminosa no estabelecimento de plântulas de $D$. pedicellatus. O primeiro experimento foi conduzido no campo, o qual teve como objetivo avaliar a variação anual da abertura do dossel e a sobrevivência nos locais onde ocorreram formações de bancos de plântulas. O segundo experimento foi conduzido em ambiente de casa de vegetação com o objetivo de avaliar os efeitos de diferentes níveis de sombreamento artificial na fotossíntese e no crescimento de plantas jovens de $D$. pedicellatus.

\section{Material e Métodos}

Sobrevivência de plântulas em ambiente florestal

O estudo foi conduzido na RPPN Serra do Teimoso, localizada no município de Jussari

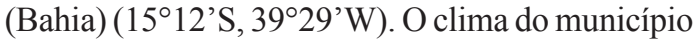
de Jussari é do tipo Am na classificação de Köppen (Bahia 2001). A sazonalidade climática é marcada por um período chuvoso que se estende de janeiro a março e um período seco de junho a dezembro. A partir de março de 2005 foi observada a formação de dois grandes bancos de plântulas de D. pedicellatus, com manchas de elevada densidade populacional. Os bancos de plântulas foram formados nos locais onde havia maior densidade de indivíduos adultos (entre 250 e 300 m.s.m.e entre 350 e 400 m.s.m). Na área da Reserva onde existe a maior densidade de indivíduos adultos de D. pedicellatus (Amorim et al. 2005; Thomas et al. 2009), e onde ocorreram as formações de banco de plântulas, o solo apresenta elevada fertilidade, baixa acidez e elevada capacidade de retenção de água; sendo classificado como Cambissolo Háplico Eutrófico léptico, unidade CEPEC Raso, franco argiloso, com afloramento de rochas. Em junho de 2005, nos locais onde foram identificadas as formações de bancos de plântulas, foram marcados 24 pontos amostrais, sendo 12 localizados entre 250 e 300 m.s.m. e 12 entre 350 e 400 m.s.m. Os pontos amostrais foram marcados com uma estaca de madeira e identificados de forma a permitir o acompanhamento contínuo, por meio da contagem de todas as plântulas existentes em parcelas circulares com $0,7 \mathrm{~m}$ de diâmetro $\left(\approx 0,4 \mathrm{~m}^{2}\right)$. $\mathrm{Na}$ primeira avaliação foram contadas, em cada parcela, todas as plântulas que apresentavam entre 5 e $10 \mathrm{~cm}$ e a presença de duas folhas cotiledonares. Com o objetivo de avaliar a sobrevivência das plântulas foram realizadas recontagens aos 92, 214, 334 e 730 dias após a primeira avaliação. Aos 730 dias após a primeira coleta de dados, em junho de 2007, foi realizada uma contagem adicional do número de plântulas; nessa ocasião quase a totalidade das mesmas ainda apresentava as características descritas acima. Durante todo o período de observações não houve nenhum outro evento de floração.

Em cada ponto amostral foram obtidas fotografias do dossel com uma câmera digital Nikon Coolpix 4300, equipada com uma lente hemisférica $\left(180^{\circ}\right)$, montada sobre um tripé a $1,30 \mathrm{~m}$ de altura e 
nivelada em relação ao solo por meio de um nível de bolha. As fotografias foram obtidas sempre no início da manhã, com a finalidade de evitar a superexposição. As fotografias foram analisadas no programa GLA (Gap Light Analyser - Frazer et al. 1999), visando quantificar a porcentagem de abertura de dossel (CO), calculada diretamente a partir da soma das frações das aberturas totais do hemisfério (fração ou porcentagem do céu visível) (Trichon et al. 1998). As avaliações de CO foram realizadas ao longo de um ano, nos mesmos dias que foram realizadas as contagens do número de plântulas.

Entre junho de 2005 e junho de 2007, dados de precipitação pluviométrica foram coletados diariamente e transformados em totais mensais, utilizando-se um pluviômetro (Meteoro Instrumentos, MG, Brasil) instalado na sede da Fazenda Teimoso. Os somatórios da precipitação pluviométrica ao longo do primeiro e do segundo ano de coleta de dados (junho de 2005 a maio de 2006 e junho de 2006 a junho de 2007) foram 975 e $1366 \mathrm{~mm}$, respectivamente. Durante o primeiro ano, valores acima de $100 \mathrm{~mm}$ foram obtidos nos meses novembro e dezembro de 2005, e janeiro, março e abril de 2006. Durante esse mesmo período, os menores valores de precipitação ocorreram entre os meses de junho e outubro de 2005. Ao longo do primeiro ano, os meses que apresentaram o maior e menor valor de precipitação pluviométrica foram abril e maio de 2006 ( 170 e 9 mm, respectivamente).

Os efeitos dos locais e da época de coleta de dados em $\mathrm{CO}$, na densidade de plântulas e na sobrevivência foram analisados mediante análise da variância seguida do teste de Tukey em nível de $5 \%$ de probabilidade. Para tal, foi adotado um delineamento experimental inteiramente casualizado, em esquema fatorial (locais $\times$ épocas), com 12 repetições. Os resultados de $\mathrm{CO}$ e de sobrevivência foram transformados para arco-seno $(\mathrm{x} / 100)^{0,5}$.

\section{Crescimento e características}

fotossintéticas em ambiente de casa

de vegetação

$\mathrm{O}$ experimento foi conduzido na casa de vegetação da UESC, localizada no município de Ilhéus, BA. Para a realização desse experimento foram coletadas plântulas originadas de regeneração natural numa área de mata secundária em estágio avançado de regeneração, localizada próxima da reserva, na Fazenda Teimoso, Jussari, Bahia. Esse procedimento foi adotado com o objetivo de evitar alterações na regeneração natural dentro da área da RPPN. As plântulas foram coletadas em julho de 2005 e apresentavam as mesmas características descritas acima para o estudo de sobrevivência, ou seja, 5 e $10 \mathrm{~cm}$ de altura e a presença de apenas duas folhas cotiledonares. As plântulas foram levadas para a casa de vegetação e transplantadas para sacos plásticos com $0,5 \mathrm{~L}$ de capacidade contendo solo florestal, onde permaneceram por um período de oito meses. Após o período de aclimatação, as plantas foram transplantadas novamente para baldes plásticos com $10 \mathrm{~L}$ de capacidade e submetidas à quatro níveis de radiação luminosa, em condições de casa de vegetação, por 289 dias. O sombreamento artificial foi obtido colocando-se as plantas nas condições naturais de radiação no interior da casa de vegetação e sob cobertura com tela preta $(25,50$ e $75 \%$ de atenuação da radiação solar), com dimensões de $2,5 \times 1,5 \times 1,5 \mathrm{~m}$. Os percentuais de atenuação da radiação solar em cada ambiente de luz foram calculados conforme descrito em Feijó et al. (2009) e corresponderam, respectivamente, a 25\% (condições naturais de radiação no interior da casa de vegetação), $17 \%, 10 \%$ e $5 \%$ com relação ao pleno sol.

No início do experimento, 10 plantas foram utilizadas para análise da biomassa no tempo zero e foram colocadas 12 plantas sob cada nível de radiação luminosa. Após 289 dias do início do experimento, todas as plantas foram coletadas para análise da massa seca de folhas (MSF), caules (MSC), raízes (MSR) e total (MST), e da área foliar total por planta (AF). A massa seca foi obtida por meio de secagem numa estufa de circulação forçada de ar a $75^{\circ} \mathrm{C}$, até que a massa atingisse valor constante. A área das folhas foi estimada por meio de um medidor de área foliar LI-3100 (Li-Cor, USA). A partir dos dados de massa seca e de área foliar foram calculadas a massa foliar por unidade de área $(\mathrm{MFE}=\mathrm{MSF} / \mathrm{AF})$, a razão de massa de raízes $(\mathrm{RMR}=\mathrm{MSR} / \mathrm{MST})$, a razão de massa de folhas $(\mathrm{RMF}=\mathrm{MSF} / \mathrm{MST})$ e a razão de área foliar $(\mathrm{RAF}=$ AF/MST). Com base nos dados de massa seca e de área foliar, foram calculadas as taxas de crescimento relativo (TCR) e assimilatória liquida (TAL), além da massa foliar específica (MFE). A TCR foi calculada pela equação TCR $=\left(\operatorname{lnM}_{2}-\ln _{1}\right) /\left(T_{2}-T_{1}\right)$, enquanto a TAL foi calculada pela equação $\mathrm{TAL}=$ $\left[\left(\mathrm{M}_{2}-\mathrm{M}_{1}\right) /\left(\mathrm{T}_{2}-\mathrm{T}_{1}\right) \times\left(\ln \mathrm{A}_{2}-\ln \mathrm{A}_{1}\right) /\left(\mathrm{A}_{2}-\mathrm{A}_{1}\right)\right] ;$ onde $\mathrm{M}_{1}$ e $\mathrm{M}_{2}$, e $\mathrm{A}_{1}$ e $\mathrm{A}_{2}$ correspondem às massas secas e às áreas foliares no início e no final do experimento, respectivamente; e $\mathrm{T}_{2}-\mathrm{T}_{1}$ correspondem ao período de tempo de aplicação dos tratamentos, em dias (Hunt 1982). 
Após 230 dias do início do experimento foram estimadas variáveis derivadas de curvas de saturação da fotossíntese pela radiação luminosa utilizando-se um sistema portátil para medição de fotossíntese LI-6400 (Li-Cor, USA), equipado com uma fonte de luz 6400-02B RedBlue. As medições foram realizadas em uma folha completamente expandida e madura por planta, utilizando-se três plantas por tratamento. As curvas foram obtidas utilizando-se o programa "light curve" do software OPEN 3.4, em sete valores de RFA 0, 15, 25, 50, $100,200,400,800,1000,1200$ e $1600 \mu$ mol fótons $\mathrm{m}^{-2} \mathrm{~s}^{-1}$, em ordem decrescente. Para cada repetição estimou-se a eficiência quântica aparente (a) e a taxa de respiração no escuro $\left(R_{d}\right)$ por meio de um modelo linear do tipo $A=a R F A+R_{d}$, para $R F A<100$ $\mu \mathrm{mol} \mathrm{m} \mathrm{m}^{-2} \mathrm{~s}^{-1}$. Esse modelo permitiu ainda estimar o valor da irradiância de compensação ( $\mathrm{IC}=\mathrm{Rd} / \mathrm{a})$, que corresponde ao ponto onde a reta toca o eixo $\mathrm{x}$. A taxa fotossintética líquida em saturação de radiação luminosa $\left(\mathrm{A}_{\max }\right)$ foi estimada por meio do ajuste um modelo exponencial do tipo $\mathrm{A}=$ $\operatorname{Amax}\left(1-\mathrm{e}^{(-\mathrm{k}(\mathrm{RFA}-\mathrm{Ic})}\right)$, onde, $\mathrm{k}$ é uma constante de proporcionalidade associada à concavidade da curva (Prado et al. 1994).

Para as variáveis de crescimento, foi adotado o delineamento experimental totalmente ao acaso, com quatro níveis de disponibilidade de radiação luminosa e 12 repetições. Os resultados obtidos foram submetidos à análise da variância, seguida do teste de Tukey em nível de $5 \%$ de probabilidade. Para as variáveis derivadas das curvas de saturação de radiação luminosa, adotou-se o delineamento experimental completamente ao acaso com dois níveis de radiação luminosa e três repetições. Os efeitos dos tratamentos foram comparados por meio da análise da variância.

\section{Resultados}

\section{Sobrevivência de plântulas em ambiente florestal}

Os valores de CO mantiveram-se, em média, maiores no Local 2, com exceção do mês de janeiro de 2006, quando a CO foi maior no Local 1 (Tab. 1). A abertura do dossel foi significativamente superior no Local 2 em relação ao Local 1 nas campanhas realizadas em junho e setembro de $2005(P<0,05$; Tukey), não diferindo significativamente entre os dois locais em janeiro e maio de $2006(P>0,05$; Tukey). Os valores máximos e mínimos para $\mathrm{CO}$ foram obtidos no Local 2, em setembro de 2005 e em maio de 2006 (10,0 e 2,7\%, respectivamente); sendo que o maior valor médio, considerando os dois locais, foi observado em setembro de 2005 (6,2\%).

Os valores médios da densidade de plântulas de $D$. pedicellatus ao longo de todo o período de coleta de dados foram estatisticamente semelhantes (ANOVA, $\mathrm{F}=0,06, \mathrm{gl}=1, P>0,10$ ) nos dois locais onde foram realizadas amostragens (Tab. 1). Pelo fato de não ter ocorrido diferenças significativas entre locais a relação entre densidade de plântulas e o tempo decorrido desde a primeira avaliação é apresentada de forma unificada na Fig. 1. Podese observar que houve uma queda abrupta na densidade de plântulas nas campanhas realizadas em junho de 2005 e janeiro de 2006. Em junho de 2005 , os valores da densidade de plântulas variaram entre 23 e 918 plântulas $\mathrm{m}^{-2}$. Já em maio de 2006, esses valores variaram entre 10 e 410 plântulas $\mathrm{m}^{-2}$. Em junho de 2007, ou seja, dois anos após o início das observações os valores da densidade de plântulas variaram entre 5 e 190 plântulas $\mathrm{m}^{-2}$; embora o valor médio de 53 plântulas $\mathrm{m}^{-2}$ ainda possa ser considerado relativamente alto. De forma semelhante ao observado para a densidade de plântulas, não foram verificadas diferenças significativas para a sobrevivência (ANOVA, F

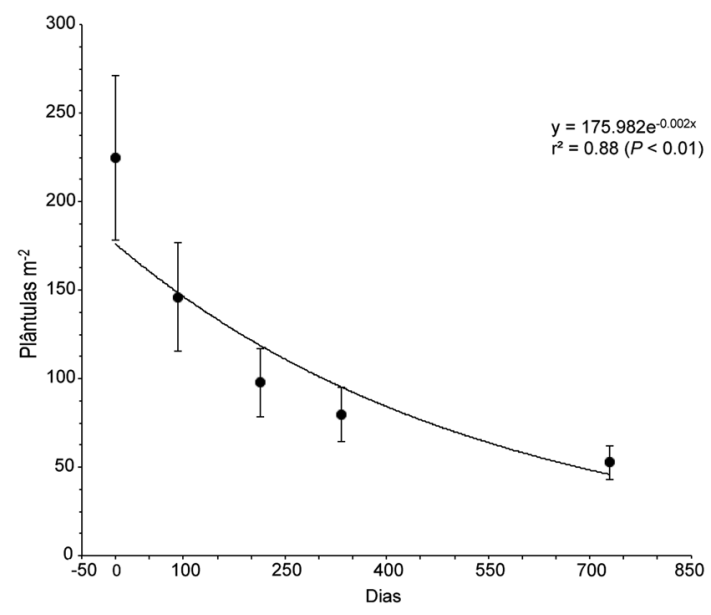

Figura 1 - Relação entre o tempo decorrido desde a primeira até a última avaliação (junho de 2005 e junho de 2007) e a densidade de plântulas de $D$. pedicellatus por unidade de área na RPPN Serra do Teimoso, Jussari, BA.

Figure 1 - Relationship between the time elapsed from the first to the last evaluation (June 2005 and June 2007) and $D$. pedicellatus seedlings density per unit area in the RPPN Serra do Teimoso, Jussari, BA, Brazil. 
Tabela 1 - Valores médios ( \pm erro padrão) da abertura do dossel $(\mathrm{CO})$, da densidade de plântulas e do percentual de sobrevivência de D. pedicellatus em junho de 2005, Setembro de 2005, Janeiro de 2006, Maio de 2006 e Junho de 2007 na RPPN Serra do Teimoso, Jussari, BA.

Table 1 - Mean values ( \pm standard error) of the canopy openness $(\mathrm{CO})$, the seedlings density and the percentage of survival of $D$. pedicellatus in June 2005, September 2005, January 2006, May 2006 and June 2007 in the RPPN Serra do Teimoso, Jussari, BA, Brazil.

\begin{tabular}{lllll|}
\hline Variável & Época & Local 1 & Local 2$^{\mathbf{( 1 )}}$ & Média $^{(\mathbf{2})}$ \\
\hline CO (\%) & Jun/05 & $3,9 \pm 0,2^{\mathrm{bB}}(3,4-4,8)$ & $6,4 \pm 0,4^{\mathrm{aA}}(4,2-8,1)$ & $5,2 \pm 0,3^{\mathrm{b}}$ \\
& Set/05 & $5,5 \pm 0,3^{\mathrm{abB}}(4,5-8,4)$ & $6,9 \pm 0,4^{\mathrm{aA}}(5,4-10,0)$ & $6,2 \pm 0,3^{\mathrm{a}}$ \\
& Jan/06 & $4,6 \pm 0,2^{\mathrm{abA}}(3,6-5,3)$ & $3,9 \pm 0,2^{\mathrm{bA}}(2,4-4,9)$ & $4,2 \pm 0,2^{\mathrm{c}}$ \\
& Mai/06 & $3,8 \pm 0,1^{\mathrm{bA}}(3,1-4,9)$ & $4,4 \pm 0,3^{\mathrm{bA}}(2,7-6,9)$ & $4,1 \pm 0,2^{\mathrm{c}}$ \\
\hline Densidade (plântulas $\left.\mathrm{m}^{-2}\right)$ & Jun/05 & $233 \pm 80^{\mathrm{aA}}(40-918)$ & $217 \pm 52^{\mathrm{aA}}(23-578)$ & $225 \pm 46^{\mathrm{a}}$ \\
& Set/05 & $151 \pm 54^{\mathrm{abA}}(23-608)$ & $141 \pm 33^{\mathrm{abA}}(20-360)$ & $146 \pm 31^{\mathrm{ab}}$ \\
& Jan/06 & $99 \pm 32^{\mathrm{bA}}(20-410)$ & $96 \pm 23^{\mathrm{bA}}(10-273)$ & $98 \pm 19^{\mathrm{b}}$ \\
& Mai/06 & $80 \pm 25^{\mathrm{bA}}(15-325)$ & $80 \pm 19^{\mathrm{bA}}(10-220)$ & $80 \pm 15^{\mathrm{b}}$ \\
& Jun/07 & $54 \pm 15^{\mathrm{bA}}(13-190)$ & $51 \pm 14^{\mathrm{bA}}(5-133)$ & $53 \pm 10^{\mathrm{b}}$ \\
\hline Sobrevivência (\%) & Set/05 & $64 \pm 3^{\mathrm{aA}}(38-79)$ & $68 \pm 4^{\mathrm{aA}}(45-79)$ & $66 \pm 3^{\mathrm{a}}$ \\
& Jan/06 & $50 \pm 4^{\mathrm{bA}}(27-79)$ & $45 \pm 3^{\mathrm{bA}}(24-56)$ & $47 \pm 3^{\mathrm{b}}$ \\
& Mai/06 & $43 \pm 5^{\mathrm{bA}}(19-79)$ & $37 \pm 3^{\mathrm{bA}}(20-48)$ & $40 \pm 3^{\mathrm{b}}$ \\
& Jun/07 & $33 \pm 5^{\mathrm{cA}}(12-69)$ & $23 \pm 4^{\mathrm{cA}}(8-44)$ & $28 \pm 3^{\mathrm{c}}$ \\
\hline
\end{tabular}

Médias seguidas pelas mesmas letras não diferem entre si em nível de 5\% de probabilidade. Letras maiúsculas indicam comparações entre locais pela análise da variância e letras minúsculas indicam comparação entre épocas pelo teste de Tukey. Valores entre parênteses correspondem aos mínimos e máximos observados. ${ }^{(1)} \mathrm{n}=12 ;{ }^{(2)} \mathrm{n}=24$

$=2,16, \mathrm{gl}=1, P>0,10)$ entre os dois locais de coleta de dados (Tab. 1). Verificou-se diferença significativa $(P<0,05$; Tukey) na sobrevivência de plântulas entre setembro de 2005 e janeiro de 2006 , com uma queda de $66 \%$ para $47 \%$. Dois anos após o início da coleta de dados as porcentagens de sobrevivência de plântulas ainda estiveram acima de $20 \%$, sendo esse valor significativamente inferior $(P<0,05$; Tukey) ao valor encontrado em maio de 2006 (40\%).

\section{Crescimento e características}

fotossintéticas em ambiente de casa de vegetação

Foram observadas diferenças significativas entre os níveis de luz para Amax $(P<0,05$; ANOVA), Rd e Ic ( $P<0,01$; ANOVA) (Tab. 2). Embora tenha ocorrido um pequeno aumento na $\alpha$ entre $25 \%$ e $5 \%$ do pleno sol, não houve diferença significativa entre tratamentos. Os valores de Amax, Rd e Ic foram, respectivamente; 1,5, 11,3 e 4,5 vezes maiores sob $25 \%$ do que sob $5 \%$ do pleno sol (Tab. 2, Fig. 2). Visto que não houve diferença significativa entre tratamentos para $\alpha$, as mudanças em Ic, conforme aumentava o sombreamento, foram diretamente relacionadas com alterações na taxa de metabolismo, demonstradas pela diminuição da Rd.
Os valores médios da RMR, MFE, TCR e TAL diminuíram e os valores médios da RMF e RAF aumentaram significativamente $(P<0,05$; Tukey) com o aumento dos níveis de sombreamento (Tab. 3). A TCR e a TAL foram, respectivamente, 1,9 e 2,7 vezes maiores sob $25 \%$ do que sob $5 \%$ do pleno sol, corroborando com os dados obtidos para as curvas de saturação de radiação luminosa (Tab. 2, Fig. 2). Por outro lado, a RMF e RAF foram, respectivamente, 1,2 e 1,8 vezes maiores sob 5\% do que sob $25 \%$ do pleno sol.

\section{Discussão}

Os valores médios de CO nos locais onde foram demarcados os pontos amostrais (em torno de 7\%) indicam características de uma floresta madura, com dossel bastante fechado (Trichon et al. 1998; Martins \& Rodrigues 2002; Nascimento et al. 2007). Nos meses de junho e setembro de 2005 foram verificados maiores valores de abertura no dossel do que nos meses de janeiro e março de 2006, demonstrando a influência da baixa precipitação pluviométrica entre junho e outubro de 2005 na deciduidade da vegetação arbórea da reserva. Os valores de $\mathrm{CO}$ indicaram que $D$. pedicellatus apresenta regeneração natural em ambientes de mata fechada, com ausência de médias e grandes clareiras. 
Tabela 2 - Taxa fotossintética líquida em saturação luminosa (Amax), taxa de respiração no escuro (Rd), eficiência quântica aparente $(\alpha)$ e irradiância de compensação (Ic) para plantas de D. pedicellatus após 230 dias crescendo em condições de casa de vegetação sob 25 e 5\% da radiação luminosa disponível a pleno sol. Médias de três repetições \pm erro padrão. Table 2 - Light saturated net photosynthetic rate (Amax), dark respiration rate (Rd), apparent quantum efficiency $(\alpha)$ and compensation irradiance (Ic) for D. pedicellatus seedlings 230 days after growing in greenhouse conditions at 25 and $5 \%$ of the light radiation available in full sun. Mean \pm standard error of three replications.

\begin{tabular}{|c|c|c|c|c|}
\hline \% Pleno sol & $\operatorname{Amax}\left(\mathrm{mmol} \mathrm{CO}_{2} \mathrm{~m}^{-2} \mathbf{s}^{-1}\right)$ & $\mathrm{Rd}\left(\mathrm{mmol} \mathrm{CO}{ }_{2} \mathrm{~m}^{-2} \mathrm{~s}^{-1}\right)$ & $\alpha\left(\mathrm{mmol} \mathrm{CO}_{2} \mathrm{mmol}\right.$ fótons $\left.^{-1}\right)$ & Ic $\left(\mathrm{mmol}\right.$ fótons $\left.\mathrm{m}^{-2} \mathrm{~s}^{-1}\right)$ \\
\hline 25 & $6,38 \pm 0,30^{*}$ & $0,34 \pm 0,04 * *$ & $0,057 \pm 0,003 \mathrm{~ns}$ & $5,96 \pm 0,43 * *$ \\
\hline 5 & $4,37 \pm 0,37$ & $0,03 \pm 0,06$ & $0,059 \pm 0,003$ & $1,32 \pm 0,27$ \\
\hline
\end{tabular}

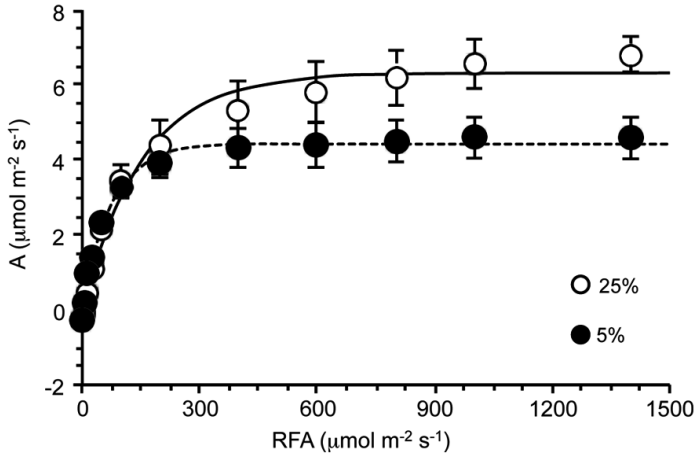

Figura 2 - Curvas de resposta da taxa fotossintética por unidade de área foliar (A) em relação à radiação fotossinteticamente ativa (RFA) para plantas de $D$. pedicellatus após 230 dias crescendo em condições de casa de vegetação sob 25 e 5\% da radiação luminosa disponível a pleno sol. Médias de três repetições \pm erro padrão.

Figure 2-Response curves of the photosynthetic rate per unit leaf area (A) to photosynthetic active radiation (RFA) for $D$. pedicellatus seedlings 230 days after growing in greenhouse conditions at 25 and $5 \%$ of the light radiation in full sun. Mean \pm standard error of three replications.

A regeneração natural de espécies florestais é dependente de inúmeras condições que variam de uma espécie para outra (Moraes \& Paoli 1999; Comita \& Hubbel 2009; Li et al. 2009; Marimon et al. 2012); porém, é sempre indispensável uma quantidade suficiente de sementes viáveis e condições edafoclimáticas que satisfaçam as necessidades de germinação e crescimento (Lamprecht 1990). No ciclo de vida de uma planta, as fases de germinação e de estabelecimento de plântulas são as mais críticas, onde normalmente ocorrem altas taxas de mortalidade (Moraes \& Paoli 1999; Comita \& Hubbel 2009). A diminuição gradual na densidade de plântulas entre os meses de setembro de 2005 e janeiro de 2006 pode ser resultado dos baixos valores da precipitação pluviométrica verificados entre junho e outubro de 2005. Assim como ocorreu no presente estudo, Moraes \& Paoli (1999) também observaram uma queda na sobrevivência de plântulas de C. moschata no período mais seco. Embora não tenham sido realizadas análises dos teores de umidade do solo ao longo do período de coleta de dados, este fator também poderia explicar, pelo menos em parte, o aumento da mortalidade nos períodos de maior déficit hídrico.

Diversas características morfológicas, anatômicas e fisiológicas diferenciam a capacidade de adaptação e aclimatação das diferentes espécies vegetais a condições de sombra ou pleno sol (Valladares \& Niinemets 2008; Poorter 1999; Poorter 2009). Folhas de sombra são em geral menos espessas, apresentam menores valores médios para $\mathrm{A}_{\max }$, Rd, Ic e MFE do que folhas de sol (Lüttge 2008). Tais características podem variar dentro de uma mesma espécie, ou entre espécies e grupos ecológicos, e estão diretamente relacionadas com a capacidade de sobrevivência e de crescimento em ambientes de clareiras ou de matas fechadas (Kitajima 1994; Barker et al. 1997). Os valores obtidos para as variáveis derivadas das curvas de saturação de radiação luminosa permitem concluir que $D$. pedicellatus apresenta características de uma espécie arbórea clímax, que regenera em ambientes sombreados e apresenta crescimento muito lento, corroborando os resultados obtidos em condições de campo. Além disso, as maiores diferenças entre os tratamentos ocorreram para Rd e Ic. Uma vez que não houve diferença significativa entre tratamentos para $\alpha$, a diminuição na Ic e a manutenção de um balanço de carbono positivo em nível foliar nos ambientes com baixa disponibilidade de radiação luminosa parecem ser dependentes da baixa atividade metabólica, demonstrada pelos baixos valores de Rd.

Ao final do período de coleta de dados, praticamente todas as plântulas localizadas nas parcelas amostrais ainda apresentavam as mesmas características morfológicas observadas na primeira avaliação; sendo muito raras aquelas 
Tabela 3 - Razão de massa de raízes (RMR) e de folhas (RMF), massa foliar específica (MFE), razão de área foliar (RAF), taxa de crescimento relativo (TCR) e taxa assimilatória líquida (TAL) de mudas de D. pedicellatus submetidas à quatro níveis de disponibilidade de radiação luminosa $(25,17,10$ e 5 \% com relação ao pleno sol), em condições de casa de vegetação, por 289 dias. Médias de 12 repetições \pm erro padrão.

Table 3 - Roots (RMR) and leaves mass ratios (RMF), leaf mass per area (MFE), leaf area ratio (RAF), relative growth rate (TCR) and net assimilation rate (TAL) of D. pedicellatus seedlings subjected to four levels of available light radiation $(25,17,10$ and $5 \%$ in relation to full sun), under greenhouse for 289 days. Mean \pm standard error of 12 replications.

\begin{tabular}{lcccc}
\hline & \multicolumn{4}{c}{$\mathbf{\%}$ Pleno sol } \\
\cline { 2 - 5 } Variável & $\mathbf{2 5}$ & $\mathbf{1 7}$ & $\mathbf{1 0}$ & $\mathbf{5}$ \\
\hline RMR & $0,26 \pm 0,02 \mathrm{a}$ & $0,22 \pm 0,2 \mathrm{ab}$ & $0,20 \pm 0,02 \mathrm{ab}$ & $0,16 \pm 0,007 \mathrm{~b}$ \\
RMF & $0,53 \pm 0,02 \mathrm{~b}$ & $0,55 \pm 0,02 \mathrm{~b}$ & $0,55 \pm 0,01 \mathrm{~b}$ & $0,62 \pm 0,01 \mathrm{a}$ \\
MFE $\left(\mathrm{g} \mathrm{m}^{-2}\right)$ & $116,11 \pm 6,73 \mathrm{a}$ & $99,36 \pm 4,25 \mathrm{ab}$ & $83,82 \pm 4,3 \mathrm{bc}$ & $75,89 \pm 2,53 \mathrm{c}$ \\
RAF $\left(\mathrm{cm}^{2} \mathrm{~g}^{-1}\right)$ & $46,7 \pm 2,8 \mathrm{c}$ & $56,5 \pm 3,4 \mathrm{bc}$ & $67,3 \pm 4,2 \mathrm{~b}$ & $82,8 \pm 3,5 \mathrm{a}$ \\
TCR $\left(\mathrm{mg} \mathrm{g}^{-1} \mathrm{dia}^{-1}\right)$ & $8,48 \pm 0,41 \mathrm{a}$ & $8,19 \pm 0,34 \mathrm{a}$ & $5,24 \pm 0,40 \mathrm{~b}$ & $4,40 \pm 0,43 \mathrm{~b}$ \\
TAL $\left(\mathrm{mg} \mathrm{cm}^{2} \mathrm{dia}^{-1}\right)$ & $0,16 \pm 0,01 \mathrm{a}$ & $0,14 \pm 0,01 \mathrm{a}$ & $0,08 \pm 0,01 \mathrm{~b}$ & $0,06 \pm 0,01 \mathrm{~b}$ \\
\hline Médias seguidas pelas mesmas letras não diferem entre si pelo teste de Tukey em nível de $5 \%$ de probabilidade.
\end{tabular}

que já haviam emitido a primeira folha verdadeira (dados não apresentados). Esse fato parece estar relacionado com a baixa disponibilidade de radiação luminosa no sub-bosque, conforme pode ser observado pelos baixos valores de $\mathrm{CO}$ nos locais onde ocorreram formações de bancos de plântulas. Por outro lado, as plântulas transplantadas para condições de casa de vegetação rapidamente lançaram folhas novas e os valores médios de TCR, TAL, $A_{\max }$ e Rd aumentaram significativamente entre os tratamentos de $5 \%$ e $25 \%$ do pleno sol; indicando uma estratégia na qual os indivíduos regenerantes conseguem responder ao aumento na disponibilidade de radiação luminosa causada pelo surgimento de clareiras no dossel florestal.

Assim como observado para as variáveis derivadas de curvas de saturação de radiação luminosa, os valores de TCR e de TAL indicam um crescimento muito lento quando comparados aos valores obtidos por outras espécies arbóreas nativas da Mata Atlântica (Souza \& Válio 2003; Feijó et al. 2009). As diminuições na TCR e TAL com o aumento do sombreamento, mesmo seguidas de aumentos na RAF, podem ter sido causadas pelos baixos valores de RFA nas condições em que foi realizado o experimento. Feijó et al. (2009) relataram valores médios e máximos de RFA de 174 e $406 \mu \mathrm{mol}$ fótons $\mathrm{m}^{-2} \mathrm{~s}^{-1}$, respectivamente, para o tratamento de $25 \%$ do pleno sol, e de 31 e $79 \mu \mathrm{mol}$ fótons $\mathrm{m}^{-2} \mathrm{~s}^{-1}$, respectivamente, para o tratamento de $5 \%$ do pleno sol. Conforme pode ser observado na Figura 2, esses valores encontravam-se abaixo dos valores da irradiância de saturação obtidos para $D$. pedicellatus, indicando que a disponibilidade de radiação luminosa foi um fator limitante ao crescimento das plantas.
Por outro lado, conforme discutido anteriormente, os baixos valores de $\mathrm{Rd}$ em condições de sombra densa indicam uma baixa atividade metabólica e economia de carbono em condições em que a disponibilidade de RFA é um fator limitante para a fotossíntese. Embora não tenham sido realizadas análises das características foliares associadas a defesas contra herbívoros ou patógenos, os resultados obtidos indicam que plântulas de $D$. pedicellatus apresentam uma grande capacidade de tolerar baixos níveis de luz por meio de ajustes no metabolismo primário, de forma a manter um balanço de carbono positivo e reagir com surtos de crescimento à medida que clareiras apareçam no dossel florestal. Essa estratégia, associada aos eventos de frutificação em massa, poderia garantir o estabelecimento de populações compostas por um grande número de indivíduos e elevada densidade populacional em ambientes de florestas semidecíduas, tal qual relatado por Thomas et al. (2009).

Com base nos resultados obtidos pode-se verificar que $D$. pedicelatus regenera em condições de baixa disponibilidade de radiação luminosa, características do interior de florestas maduras com dossel fechado. O processo de regeneração de $D$. pedicellatus está baseado na formação de bancos de plântulas com elevada densidade de indivíduos por unidade de área, em alguns casos com mais de 700 plântulas $\mathrm{m}^{-2}$. A estratégia de regeneração por bancos de plântulas e a capacidade de ajustar o seu metabolismo em função da radiação luminosa disponível parecem ser os fatores importantes para o estabelecimento de populações com elevada densidade populacional nas florestas semidecíduas do sul da Bahia 


\section{Agradecimentos}

Os autores agradecem aos funcionários da RPPN Serra do Teimoso, Francisco e Edvaldo, e aos proprietários da Fazenda Teimoso, Henrique F. Berbert de Carvalho e Lucélia de Melo Berbert, o auxílio prestado na coleta de dados; às Dras Adriana M. Z. Martini e Letícia dos Anjos Silva, as valiosas sugestões durante a elaboração do manuscrito; ao pesquisador Quintino R. Araújo, as informações referentes aos solos da RPPN Serra do Teimoso; ao Núcleo de Bacias Hidrográficas da UESC, os dados fornecimento dos dados de precipitação pluviométrica; e aos revisores anônimos, as contribuições ao texto final. Esse estudo foi realizado com recursos da UESC e da Fundação de Amparo à Pesquisa do Estado da Bahia (FAPESB). Dinara A. Mercês foi bolsista de iniciação científica PROIIC/UESC, Marayana P. Pinheiro foi bolsista de mestrado da CAPES e Josafá A.Oliveira Filho foi bolsista de iniciação científica PIBIC/CNPq. Marcelo S. Mielke é bolsista de Produtividade em Pesquisa do CNPq.

\section{Referências}

Amorim, A.M.; Jardim, J.G.; Thomas, W.W.; Clifton, B.C. \& Carvalho, A.M. 2005. The vascular plants of a forest fragment in Southern Bahia, Brazil. SIDA 21: 1726-1752.

Auer, C.G. \& Graça, M.C.E. 1995. Método de produção de mudas de canela-sassafrás a partir de mudas de regeneração natural. Boletim de Pesquisa Florestal 30/31: 75-77.

Bahia. Governo do Estado. 2001. Diagnóstico das bacias hidrográficas dos rios Cachoeira e Almada. Caracterização climatológica. Vol. I, Tomo III. SEINFRA/SRH, Salvador. 80p.

Baltzer, J.L. \& Thomas, S.C. 2007. Determinants of whole-plant light requirements in Bornean rain forest tree saplings. Journal of Ecology 95: 1208-1221.

Barker, M.G.; Press, M.C. \& Brown, N.D. 1997. Photosynthetic characteristics of dipterocarp seedlings in three tropical rain forest light environments: a basis for niche partitioning. Oecologia 112: 453-463.

Chazdon, R. 1988. Sunflecks and their importance to forest understorey plants. Advances in Ecological Research 18: 1-63.

Comita, L.S. \& Hubbel, S.P. 2009. Local neighborhood and species' shade tolerance influence survival in a diverse seedling bank. Ecology 90: 328-334.

Evans, J.R. \& Poorter, H. 2001. Photosynthetic acclimation of plants to growth irradiance: the relative importance of specific leaf area and nitrogen in maximizing carbon gain. Plant, Cell and Environment 24: 755-767.
Feijó, N.S.A.; Mielke, M.S.; Gomes, F.P.; França, S. \& Lavinsky, A.O. 2009. Growth and photosynthetic responses of Gallesia integrifolia (Spreng.) Harms and Schinus terebinthifolius Raddi seedlings in dense shade. Agroforestry Systems 77: 49-58.

Fiaschi, P. \& Cordeiro, I. 2005. Discocarpus pedicellatus, a new species of Phyllanthaceae (Euphorbiaceae s.1.) from southern Bahia, Brazil. Brittonia 57: 248-251.

Frazer, G.W.; Canham, C.D. \& Lertzman, K.P. 1999. Gap Light Analyzer (GLA). Simon Fraser University, Millbrook. 36p.

Higuchi, P.; Reis, M.G.F.; Reis, G.G.; Pinheiro, A.L.; Silva, C.T. \& Oliveira, C.H.R. 2006. Composição florística da regeneração natural de espécies arbóreas ao longo de oito anos em um fragmento de floresta estacional semidecidual, em Viçosa, MG. Revista Árvore 30: 893-904.

Hunt, R. 1990. Basic growth analysis. Unwin Hyman, Londres. 112p.

Kitajima, K. 1994. Relative importance of photosynthetic traits and allocation patterns as correlates of seedling shade tolerance of 13 tropical tree species. Oecologia 98: 419-428.

Kelly, J.; Jose, S.; Nichols, J.D. \& Bristow, M. 2009. Growth and physiological response of six Australian rainforest tree species to a light gradient. Forest Ecology and Management 257: 287-293.

Kenzo, T.; Yoneda. R.; Matsumoto. Y.; Mohamad Azani, A. \& Majid, M.N. 2011. Growth and photosynthetic response of four Malaysian indigenous tree species under different light conditions. Journal of Tropical Forest Science 23: 271-281.

Lamprecht, H. 1990. Silvicultura nos trópicos. GTZ, Curitiba. 343p.

Leakey, A.D.B.; Scholes, J.D. \& Press, M.C. 2005. Physiological and ecological significance of sunflecks for dipterocarp seedlings. Journal of Experimental Botany 56: 469-482.

Li, R.; Yu, S.; Wang, Y.; Staehelim, C. \& Zang, R. 2009. Distance-dependent effects of soil-derived biota on seedling survival of the tropical tree legume Ormosia semicastrata. Journal of Vegetation Science 20: 527-534.

Lüttge, U. 2008. Physiological ecology of tropical plants. Springer, Berlin. 458p.

Marimom, B.S.; Felfili, J.M.; Fagg, C.W.; MarimomJunior, B.H.; Umetsu, R.K.; Oliveira-Santos, C.; Morandi, P.S.; Lima, H.S. \& Nascimento, A.R.T. 2012. Monodominance in a forest of Brosimum rubescens Taub. (Moraceae): structure and dynamics of natural regeneration. Acta Oecologica 43: 134-139.

Martini, A.M.Z.; FiaschiI, P.; Amorim, A.M. \& Paixão, J.L. 2007. A hot-point within a hot-spot: a high diversity site in Brazil's Atlantic Forest. Biodiversity Conservation 16: 3111-3128.

Martins, S.V. \& Rodrigues, R.R. 2002. Gap-phase regeneration in a semideciduous mesophytic forest, south-eastern Brazil. Plant Ecology 163: 51-62. 
Moraes, P.L.R. \& Paoli, A.A.S. 1999. Morfologia e estabelecimento de plântulas de Cryptocarya moschata Nees, Ocotea catharinensis Mez, Endlicheria paniculata (Spreng) MacBride - Lauraceae. Revista Brasileira de Botânica 22: 287-295.

Nascimento, A.R.T.; Fagg, J.M.F. \& Fagg, C.W. 2007. Canopy openness and LAI estimates in two seasonality deciduous forest on limestone outcrops in central Brazil using hemispherical photographs. Revista Árvore 31: 167-176.

Niinemets, U. 2010. A review of light interception in plant stands from leaf to canopy in different plant functional types and in species with varying shade tolerance. Ecological Research 25: 693-714.

Poorter, L. 1999. Growth responses of 15 rain-forest tree species to a light gradient: the relative importance of morphological and physiological traits. Functional Ecology 13: 396-410.

Poorter, L. 2009. Leaf traits show different relationships with shade tolerance in moist versus dry tropical forests. New Phytologist 181: 890-900.

Prado, C.H.B.A.; Moraes, J.A.P.V. \& Mattos, E.A. 1994. Gas exchange and leaf water status in potted plants of Copaifera langsdorffi. I. Responses to water stress. Photosynthetica 30: 207-213.

Sanches, M.C.; Mielke, M.S.; Souza, C.S.D.; Vieira, A.J.D.; Lopes, M.M.M. \& Silva Jr., M.B. 2009. Morfologia foliar de indivíduos jovens e adultos de Caesalpinia echinata Lam. numa floresta semidecídua do sul da Bahia. Revista Árvore 33: 885-893.

Souza, R.P. \& Válio, I.F.M. 2003. Seedling growth of fifteen Brazilian tropical tree species differing in successional status. Revista Brasileira de Botânica 26: $35-47$.
Thomas, W.W.; Carvalho, A.M.V.; Amorim, A.M.; Garrison, J. \& Santos, T.S. 2008. Diversity of woody plants in the Atlantic coastal forest of southern Bahia, Brazil. In: Thomas, W. (ed.). The Atlantic coastal forests of northeastern Brazil. Memoirs of the New York Botanical Garden 100: 21-66.

Thomas, W.; Carvalho, A.M.; Amorim, A.M.A.; Garrison, J. \& Arbeláez, A.L. 1998. Plant endemism in two forests in southern Bahia. Biodiversity and Conservation 7: 311-322.

Thomas, W.W.; Jardim, J.G.; Fiaschi, P.; Mariano-Neto, E. \& Amorim, A.M. 2009. Composição florística e estrutura do componente arbóreo de uma área transicional de floresta atlântica no sul da Bahia, Brasil. Revista Brasileira de Botânica 32: 65-78.

Trichon, V.; Walther, J.N. \& Laumonier, Y. 1998. Identifying spatial patterns in the tropical rain forest structure using hemispherical photographs. Plant Ecology 137: 227-244.

Valladares, F. \& Niinemets, U. 2008. Partial sunlight tolerance, a key plant feature of complex nature and consequences. Annual Review of Ecology, Evolution and Systematics 39: 237-257.

Viani, R.A.C. \& Rodrigues, R.R. 2007. Sobrevivência em viveiro de mudas de espécies nativas retiradas da regeneração natural de remanescente florestal. Pesquisa Agropecuária Brasileira 42: 1067-1075.

Walters, M.B. \& Reich. P.B. 1999. Low-light carbon balance and shade tolerance in the seedlings of woody plants: do winter deciduous and broadleaved evergreen species differ? New Phytologist 143: $143-154$ 